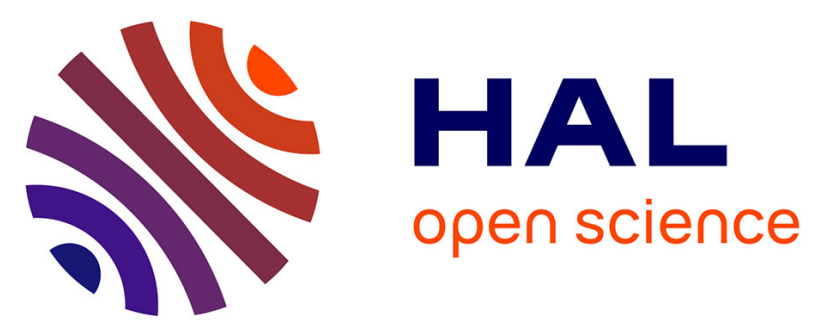

\title{
Experimental infection of pigs by Salmonella Derby, S. Typhimurium and monophasic variant of S. Typhimurium: Comparison of colonization and serology
}

María Cevallos-Almeida, Laure Martin, Catherine Houdayer, Valerie Rose, Jean-Marie Guionnet, Frédéric Paboeuf, Martine Denis, Annaëlle Kerouanton

\section{- To cite this version:}

María Cevallos-Almeida, Laure Martin, Catherine Houdayer, Valerie Rose, Jean-Marie Guionnet, et al.. Experimental infection of pigs by Salmonella Derby, S. Typhimurium and monophasic variant of S. Typhimurium: Comparison of colonization and serology. Veterinary Microbiology, 2019, 231, pp.147 - 153. 10.1016/j.vetmic.2019.03.003 . hal-03485723

\section{HAL Id: hal-03485723 \\ https://hal.science/hal-03485723}

Submitted on 20 Dec 2021

HAL is a multi-disciplinary open access archive for the deposit and dissemination of scientific research documents, whether they are published or not. The documents may come from teaching and research institutions in France or abroad, or from public or private research centers.
L'archive ouverte pluridisciplinaire HAL, est destinée au dépôt et à la diffusion de documents scientifiques de niveau recherche, publiés ou non, émanant des établissements d'enseignement et de recherche français ou étrangers, des laboratoires publics ou privés.

\section{(ㄷ)(1) $\$$}

Distributed under a Creative Commons Attribution - NonCommerciall 4.0 International 
1 Experimental infection of pigs by Salmonella Derby, $S$. Typhimurium and monophasic variant of $S$. Typhimurium: comparison of colonization and serology

3

4 María Cevallos-Almeida ${ }^{1,3}$, Laure Martin ${ }^{1}$, Catherine Houdayer ${ }^{1}$, Valerie Rose ${ }^{1}$, Jean-Marie 5 Guionnet $^{2}$, Frédéric Paboeuf ${ }^{2}$, Martine Denis $^{1}$ and Annaëlle Kerouanton ${ }^{1}$

6

$7{ }^{1}$ ANSES, Hygiene and Quality of Poultry and Pig Products Unit, Bretagne Loire University, 8 BP 53, 22440 Ploufragan, France.

92 ANSES, SPF Pig Production and Experimental Unit, Bretagne Loire University, BP 53, 1022440 Ploufragan, France.

$11{ }^{3}$ Faculty of Veterinary Medicine and Zootechnics. Central University of Ecuador. Quito, 12 Ecuador

14 Corresponding author :

15 Annaëlle Kerouanton

$16 \quad 330(2) 96018531 / 330(2) 96018538$

17 Annaelle.kerouanton@anses.fr 
Salmonella serovars Derby, Typhimurium and the monophasic variant of Salmonella Typhimurium are the most frequently isolated serovars in pigs in France. To compare the excretion patterns, seroconversion to Salmonella and contamination of the organs of pigs inoculated with strains of all three serovars, we conducted an experimental trial with 28 SPF piglets. Four were used as a negative control, while the other 24 were divided equally into three groups. Each group was inoculated at 7 weeks of age with a different strain: $S$. Derby (SDb), $S$. Typhimurium (ST), and the monophasic variant of $S$. Typhimurium (mST). Fecal and blood samples were collected twice a week up until necropsy, on 21 days post-inoculation (DPI) for half of each group and 49 DPI for the remaining piglets. During necropsy, the tonsils, mesenteric lymph nodes and various intestinal contents were collected from each pig. Salmonella bacteria were quantified in CFU/g by a bacteriological method, and levels of Salmonella antibodies were measured using an ELISA Kit. Piglets inoculated with mST continuously excreted Salmonella in their feces throughout the trial. For the other serovars, at least one piglet was Salmonella-negative on one or several DPI. The quantity of Salmonella excreted was statistically different between the group inoculated with ST and $\mathrm{mST}(\mathrm{p}<0.05)$, but no differences were found between the other serovars. The tonsils, cecum and jejunum were the most contaminated organs in all groups. Seroconversion for all the piglets was completed by different DPI: 28 for ST, 31 for mST and 38 for SDb. No major differences were found in terms of excretion and colonization among the studied serovars.

Key words: Salmonella; Typhimurium; Derby; monophasic, Pig; colonization; antibody 


\section{Introduction}

Salmonella (or S. enterica, subspecies enterica) is responsible for human and/or animal salmonellosis and is one of the most broadly-distributed foodborne pathogens worldwide. In Europe, it is the second-ranked cause of zoonosis after Campylobacter sp., with a notification rate of 21.2 cases per 100,000 population (EFSA and ECDC, 2016). In France, Campylobacter spp., Salmonella spp., and norovirus were responsible for $73 \%$ of all foodborne illnesses and 76\% of all associated hospitalizations (Van Cauteren et al., 2017). S. Typhimurium is recognized as the predominant serovar isolated from humans in Europe and $S$. Derby was one of the ten serovars most frequently isolated from humans in different countries (Boyen et al., 2008; EFSA and ECDC, 2016). The monophasic variant of Salmonella Typhimurium is an emerging serovar in human infections around the world (Switt et al., 2009; Weaver et al., 2017). Source attribution studies have established that pork has a key role in foodborne outbreaks of human salmonellosis, because in several investigations many isolates detected in pigs were responsible for these human cases (Bonardi, 2017). Pigs can be infected by several Salmonella serovars and the occurrence of these serovars is also partly geographically-determined (Boyen et al., 2008; Xu et al., 2017). S. Typhimurium, $S$. Derby, and the monophasic variant of $S$. Typhimurium are nowadays the most frequently isolated serovars from swine and pork products in the European Union (Bonardi, 2017; EFSA and ECDC, 2016; Gosling et al., 2018). In France, $S$. Derby and $S$. Typhimurium are also the most frequently isolated serovars in pig production (Denis et al., 2013) and, in the last ten years, the French Salmonella Network has observed an increase in the diagnosis of monophasic serovars in animal feed and environmental sources (Bugarel et al., 2012b).

Differences in the excretion and immune response in pigs have been reported between Salmonella serovars (Ivanek et al., 2012; Osterberg et al., 2010), but these studies have never included the monophasic variant of $S$. Typhimurium. 
While all three serovars are present in pork products prior to ingestion, serovar Typhimurium and its monophasic variant are more widely implicated in human salmonellosis. In addition, the infection produced by $S$. Derby seems to be less severe than that produced by S. Typhimurium (Ivanek et al., 2012; Matiasovic et al., 2014; Osterberg et al., 2009). To better understand the duration and dynamics of fecal shedding and seroconversion after infection with these three pig-associated serovars, we carried out an experimental trial, which was conducted with the same parameters as those previously used for an experimental trial on monophasic S. Typhimurium (Cevallos-Almeida et al., 2018).

75

2. Materials and methods

\subsection{Strains}

Three strains of Salmonella from the ANSES laboratory's private collection were used to inoculate the piglets. The strains had been isolated in pigs' fecal samples at farm level or in their mesenteric lymph nodes at the slaughterhouse (Table 1). To ensure that strains could be easily enumerated during the trial, they were made resistant to Rifampicin (Rif) after several passages on XLD agar (Biokar, France) supplemented with Rifampicin (100 mg/lt, Sigma, France). Rif strains were checked to confirm similarity to parental strains using conventional serotyping, PFGE typing (Ribot et al., 2006) and antimicrobial susceptibility testing (data not shown).

\subsection{Experimental design}

This in vivo trial protocol was approved by the Ethics Committee on Animal Research No. 16, of the French Ministry of National Education, Higher Education and Research (APAFiS license no. 7689-2016112311346069). The trial was conducted on 28 secondary specificpathogen-free (SPF) Large White piglets born at ANSES Ploufragan's protected animal 
91 facilities. The secondary SPF piglets are naturally born from SPF sows controlled for 92 presence of several pathogens (Le Devendec et al., 2018).

93 At 4 weeks of age, three weeks before inoculation, the piglets were weaned and placed in

94 separate hermetic experimental animal rooms of biosecurity level 3. The 24 piglets intended 95 to be inoculated were placed in three separate rooms, with two pens per room (eight piglets 96 per room, four per pen). Each room was considered a group. In Groups SDb, ST, and mST, 97 piglets were inoculated by $S$. Derby, $S$. Typhimurium and the monophasic variant of $S$. 98 Typhimurium respectively.

99 Staff at the animal facility complied with all the necessary biosecurity measures. The piglets 100 were monitored daily; their rectal temperature was taken and any clinical manifestations were 101 recorded. Food consumption and body weight were reported weekly.

\subsection{Experimental Inoculation}

103 Seven days before inoculation, the 28 piglets were tested and confirmed to be free of

104 Salmonella. Fecal samples from each piglet were analyzed in accordance with the NF U47105102 method (AFNOR, 2008). On day 0, the 24 piglets of seven weeks of age were inoculated 106 as previously described for an earlier experimental study (Cevallos-Almeida et al., 2018). The 107 inoculum were prepared after an overnight culture of the three strains at $37^{\circ} \mathrm{C}$. Cultures were 108 adjusted in Tryptone Salt Broth (SLT) (bioMérieux, France), after a DO measurement, in 109 order to obtain an inoculum of about $10^{8} \mathrm{CFU} / \mathrm{ml}\left(8 \log _{10} \mathrm{CFU} / \mathrm{ml}\right)$. After numeration, 110 inoculum concentrations were $8.23,8.40,8.19 \log _{10} \mathrm{CFU} / \mathrm{ml}$ respectively for SDb, STM and 111 vmST. Then, piglets were inoculated orally, using a cannula connected to a screw syringe, 112 with $10 \mathrm{ml}$ of the inoculum. The four control piglets were given $10 \mathrm{ml}$ of SLT orally. 
115 Fecal samples (at least $30 \mathrm{~g}$ ) were taken directly from the animal rectum on 1, 2 and 3 DPI in 116 the first week, and then twice a week until 49 DPI.

117 To determine seroconversion, blood samples were taken on the same dates as fecal samples.

118 The blood was then centrifuged at $3500 \mathrm{rpm}$ for 5 minutes to recover the serum in order to

119 determine anti-Salmonella antibody levels. These sera were stored at $-20^{\circ} \mathrm{C}$ until they were 120 analyzed.

121 The three groups were necropsied at different ages: 21 DPI for 14 piglets (four in each group)

122 and 49 DPI for the remaining piglets. The four control piglets were kept in a separate room 123 and also slaughtered at different ages: two piglets on 21 DPI and two piglets on 49 DPI. 124 Piglets were euthanized through intravenous injection with an overdose of tiletamine and 125 zolazepam (Zoletil 100, Virbac, France). The subsequent necropsies primarily examined 126 organs and tissues in the abdominal and thoracic cavities. The tonsils, mesenteric lymph nodes (MLN) and intestinal contents of the duodenum, jejunum, ileum and cecum were 128 collected.

\subsection{Enumeration and detection of Salmonella}

130 All the analyses were performed as previously described (Cevallos-Almeida et al., 2018).

131 Briefly, fecal and necropsy samples were diluted 1:10 in Buffered Peptone Water (BPW,

132 bioMérieux, France). Serial dilution was then performed in the SLT until a dilution of $10^{-4}$ 133 was obtained. One milliliter of the $10^{-1}$ dilution was seeded on three plates of XLD 134 supplemented with rifampicin (XLD-Rif), and $100 \mu \mathrm{l}$ of each of the subsequent dilutions was 135 plated on separate plates of XLD-Rif. After incubation at $37^{\circ} \mathrm{C}$ for 24 hours, typical black 136 colonies of Salmonella were counted to enumerate Salmonella in terms of CFU/g for each 137 sample. If enumerations for Salmonella sp. were negative due to being below the enumeration 138 limit (10 CFU/g), the NF U47-102 method (AFNOR, 2008) was used to enrich the sample so 
as to confirm the presence or absence of Salmonella in the sample. This method was also

140 applied to the samples collected from control piglets throughout the assay in order to confirm

141 the sustained absence of Salmonella.

\subsection{Antibody response to Salmonella}

143 For antibody screening, we used the IDEXX Swine Salmonella Ab Test ${ }^{\circledR}$ (IDEXX, France).

144 This indirect ELISA is based on detecting the Salmonella lipopolysaccharide (LPS) antigen.

145 In accordance with the manufacturer's recommendations, samples with an optical density

146 (OD) equal to or greater than $15 \%$ were regarded as positive.

\subsection{Data Management and statistical analysis}

148 Statistical analysis was performed using $\mathrm{R}$ software ( $\mathrm{R}$ version 3.2.4). The bacterial excretion

149 level was compared between groups for each sampling point post-inoculation with a Tukey

150 test $(\mathrm{p}<0.05)$. The CFU/g in feces from each sampling point was normalized logarithmically

151 and plotted in order to calculate the cumulative area under the log curve (AULC) to determine

152 total Salmonella shedding for each piglet over the course of the study. A Kruskal-Wallis test

153 was used to compare the AULC between groups $(\mathrm{p}<0.05)$. For the data obtained from

154 necropsy, we compared the contamination levels of each group at the two dates of necropsy

155 with a Kruskal-Wallis Test $(\mathrm{p}<0.05)$.

\section{Results}

\subsection{Clinical monitoring}

158 No clinical signs were found in the control piglets. The clinical signs displayed by the

159 inoculated piglets differed depending on the strain inoculated. Transitory diarrhea was found

160 in all groups at least once: for Group SDb on 14 DPI, for Group ST on 7 DPI and 10 DPI, and

161 for Group mST on 21 DPI. For Group mST, no fever was observed at any point during the 
trial. Furthermore, we found no statistical difference $(p>0.05)$ between inoculated piglets and

163 the control group in terms of weight or feed consumption.

\subsection{Excretion in fecal samples}

165 The control piglets remained negative for Salmonella in their feces throughout the trial. In

166 Group $\mathrm{mST}$, all the piglets were positive on all sampling days, and fecal excretion was continuous. For Group SDb, one piglet was negative on the day 21 after inoculation (Fig 2.). In Group ST, two piglets were negative; for one on the day 14 after inoculation (Fig 2) and, another one, on the day 45 after inoculation.

170 Mean excretion and standard deviation data for all groups are presented in Table 2.

171 We found a statistical difference by the Holm multiple comparison test $(\mathrm{p}<0.05)$ between the 172 average excretion levels obtained in the groups on 14, 17 and 21 DPI (Figure 1). On 14 DPI, 173 Group SDb differed from Group ST; on 17 DPI, Group SDb differed from Group mST; and 174 on 21 DPI, Group mST differed from Groups SDb and ST.

175 The inoculated piglets in Groups ST and mST euthanized on 21 DPI showed an excretion 176 peak on 1 DPI, with a variation in levels during the trial. Group SDb also showed a high 177 excretion level on 1 DPI but the peak was on 14 DPI, linked to a case of diarrhea the same 178 day (Figure 2).

179 By comparing AULC measurements from the excretion curves of the 24 piglets over 21 DPI 180 using the Kruskal-Wallis test, a significant difference ( $\mathrm{p}=0.008)$ was evident between Group $181 \quad$ ST and Group mST (Figure 3).

\subsection{Salmonella colonization in organs and intestinal contents}

183 At each necropsy date, $100 \%$ of the cecum content and tonsil samples were colonized in every

184 group. Between $25 \%$ and $75 \%$ of mesenteric lymph nodes were colonized depending on the

185 serovar and the necropsy date. For all serovars, contamination levels in intestinal contents 
186 increased through the intestinal passage up until the ileum, then began to decrease in the

187 cecum and fecal samples. For all serovars, the lowest levels of contamination were found in 188 the duodenum (0.7 to $1.3 \log _{10} \mathrm{CFU} / \mathrm{g}$ ) and mesenteric lymph nodes (0.7 to $\left.2.3 \mathrm{Log}_{10} \mathrm{CFU} / \mathrm{g}\right)$, 189 while the tonsils were the most significantly contaminated $(\mathrm{p}<0.01)$ on both necropsy days, 21 190 and 49 DPI (4.7 to $5.7 \log _{10} \mathrm{CFU} / \mathrm{g}$ ) (Table 3). On the basis of the analysis performed at 191 necropsy, we found no statistical difference between serovars, regardless of the type of 192 sample, either on day 21 or on day $49(\mathrm{p}>0.05)$.

\subsection{Antibody responses}

194 The OD\% means per day are shown in Table 1. Piglets produced detectable antibodies to the 195 Salmonella LPS antigen, indicating detectable seroconversion, on 7 DPI for piglets in Groups 196 SDb and mST, and 10 DPI for piglets in Group ST. The seroconversion of $100 \%$ of piglets 197 was completed by different dates depending on the serovar: all piglets in Group ST had 198 seroconverted by 28 DPI, piglets in Group mST by 31 DPI, and piglets in Group SDb by 38 199 DPI.

200 On 1 and 3 DPI, the bacterial excretion frequencies and levels were high in each group 201 (Figure 4) and the frequency of seroconverting piglets started to increase in all groups from 202 day 7. On day 49, $100 \%$ of piglets shedding Salmonella were shown to have seroconverted 203 through an ELISA.

\section{Discussion}

Salmonella serovars commonly associated with swine are a major human health problem. In our study we experimentally inoculated three groups of piglets with strains of three major swine serovars: $S$. Derby, $S$. Typhimurium and monophasic $S$. Typhimurium. We compared

208 excretion patterns, seroconversion and the colonization of several organs in the three groups of animals. We found differences in some clinical signs, such as diarrhea and fever in piglets. 
210 While all groups had transient diarrhea at least once, no fever was ever recorded in the mST 211 group. In other studies comparing either infection by $S$. Typhimurium versus $S$. Yoruba, or $S$.

212 Derby versus S. Cubana, no clinical signs or diarrhea were observed (Osterberg et al., 2009;

213 Osterberg and Wallgren, 2008). However, for $S$. Typhimurium, several clinical signs such as

214 fever, diarrhea and anorexia were reported in animals inoculated with a dose of $10^{9} \mathrm{CFU}$ 215 (Boyen et al., 2009).

216 The excretion dynamics for all groups showed that the highest level of excretion was reached 217 on 1 DPI, except for the group inoculated with $S$. Derby. In this group, the excretion was also 218 very high on day 1 DPI, but the maximum amount of Salmonella excreted was observed on 14 219 DPI. In the days following inoculation, Salmonella excretion varied depending on the serovar 220 inoculated. Only piglets in the $\mathrm{mST}$ group were shown to shed Salmonella continuously 221 throughout the experimental trial. In the SDb group, one piglet was negative for Salmonella 222 on one day of sampling 21 DPI, but he was then necropsied. At necropsy, their tonsils, ileum 223 and caecum were Salmonella positive. For ST group, two pigs had feces salmonella-free, one 224 on 14 DPI and the other on 45 DPI. The two pigs became again salmonella shedders at the 225 following sampling dates, indicating intermittent shedding.

226 By comparing total shedding (AULC), we found statistical differences between piglets 227 inoculated with serovars $S$. Typhimurium and those inoculated with the monophasic variant of 228 S. Typhimurium. The monophasic variant strain caused a higher excretion level than the 229 S. Typhimurium strain. This study is the first comparison of colonization by $S$. Typhimurium 230 versus that by monophasic $S$. Typhimurium. Even though the monophasic variant is 231 genetically related to $S$. Typhimurium (Bugarel et al., 2012a; Soyer et al., 2009; Vieira-Pinto 232 et al., 2012), further research appears necessary to establish the differences between these two 233 serovars in their colonization of pigs. 
234 On the other hand, no difference was observed between Sdb and ST or between Sdb and mST.

235 In 2012, Ivanek noted that pigs inoculated with the pig-associated serovars $S$. Typhimurium

236 and $S$. Derby tend to shed Salmonella in shorter periods of time (both as part of continuous

237 and intermittent shedding) than pigs inoculated with the feed-associated serovars $S$. Yoruba

238 and $S$. Cubana detected in Sweden (Ivanek et al., 2012). However, pigs infected with $S$.

239 Typhimurium and $S$. Derby were more likely to (re-)enter an intermittent non-shedding state.

240 Consequently, they showed a tendency towards longer-lasting host infection overall, with a

241 similar or longer total duration of fecal shedding.

242 A recent longitudinal study in pigs naturally infected with Salmonella Typhimurium indicates 243 that this serovar may also cause high shedding rates among colonized pigs in natural

244 conditions, particularly in younger animals (Weaver et al., 2017).

245 At necropsy, on 21 and 49 DPI, the tonsils and cecum of all the inoculated piglets were 246 systematically found to be contaminated by one of the three Salmonella serovars. A previous 247 study demonstrated that several serovars can contaminate the tonsils and cecum 3 hours after 248 inoculation (Loynachan, 2004). In 2004, Cote et al. also reported 100\% colonization for the 249 tonsils, cecum and MLN on 14 DPI (Cote et al., 2004). On average, the occurrence of positive 250 samples was the lowest in MLN, then in the contents of the duodenum. The jejunum and 251 ileum were more highly colonized, with $87.5 \%$ and $95.8 \%$ respectively. These observed 252 trends are the same regardless of the serovar used for inoculation. Matiasovic et al. (2014) 253 showed that after $S$. Typhimurium inoculation, only the tonsils and lymph nodes remained 254 culture-positive up until 28 DPI and only the tonsils and cecum remained culture-positive 255 following inoculation with $S$. Derby. In 2010, Osterberg et al. compared direct and indirect 256 transmission of $S$. Derby and $S$. Typhimurium. For indirect transmission, pigs were 257 necropsied after 14 days in a contaminated environment. For $S$. Derby, the cecum content, 258 colon tissue and ileocecal lymph node were found to be contaminated in only one pig out of 
six $(16 \%)$. For $S$. Typhimurium, four out of six of the pigs $(66 \%)$ were culture-positive for

260 cecum and/or ileocecal lymph node samples. In terms of data for the monophasic variant, in a 261 previous study we found similar results for the colonization of tonsils and intestinal contents, 262 but MLNs were more highly contaminated, with $100 \%$ and $62.5 \%$ of the samples positive on 263 21 and 49 DPI respectively (Cevallos-Almeida et al., 2018). In 2004, Loynachan detected that $26475 \%$ of tonsils were culture-positive 3 hours after inoculation with the monophasic variant 265 (Loynachan et al., 2004). Tonsils are also a common site of contamination at slaughter, with 266 about 10\% contaminated with Salmonella (Van Damme et al., 2018; Vieira-Pinto et al., 2005), and are a major source of carcass contamination, along with the contents of the intestinal tract (Berends et al., 1997).

269 For all serovars, the highest level of contamination in organs and intestinal contents was 270 found in the tonsils, cecum and jejunum. The least contaminated sites were the lymph nodes 271 and the duodenum. In 2008, Scherer et al. found similar amounts of Salmonella to those we 272 discovered in the tonsils (4.4 $\left.\log _{10} \mathrm{CFU} / \mathrm{g}\right)$ and ileum (2.7 $\left.\log _{10} \mathrm{CFU} / \mathrm{g}\right)$ after inoculation with 273 S. Typhimurium (Scherer et al., 2008). In a recent study in which pigs were inoculated with $2741 \times 10^{9} \mathrm{CFU}$ of a multidrug-resistant monophasic S. Typhimurium strain, Shippy et al. made 275 contrasting observations after 7 DPI. Specifically, tissues including the ileocecal lymph nodes 276 were more highly contaminated than cecal content, and the tonsils were the least 277 contaminated organ, with a mean of $1 \log _{10} \mathrm{CFU} / \mathrm{g}$ (Shippy et al., 2018).

278 In our study, piglets seroconverted at different times depending on the serovar inoculated. We 279 found $100 \%$ of seroconversion in group mST piglets by $31 \mathrm{DPI}$. Our results are similar to 280 those of Lynch et al., who found that $85 \%$ of pigs had seroconverted by 28 DPI (Lynch et al., 281 2017). For piglets in groups ST and SDb, seroconversion was $100 \%$ completed by 28 and 38 282 DPI. Matiasovic et al. (2014) previously described a lower immune response after infection 283 with $S$. Derby than with $S$. Typhimurium. Another study revealed a different result, with 
284 seroconversion of $100 \%$ of pigs within two weeks of infection (Osterberg and Wallgren, 285 2008). For $S$. Derby our results are different to those found by Osterberg et al. (2009), with $286100 \%$ seroconversion after 21 days.

288 This study revealed similar profiles of Salmonella excretion and colonization regardless of the 289 serovar used for experimental inoculation. The amount of excretion, however, was different 290 between groups inoculated with $S$. Typhimurium 07CR095 and monophasic $S$. Typhimurium 291 S12AK50. Seroconversion had occurred by different days depending on the serovar used for 292 inoculation. Further research is necessary to establish the virulence gene patterns of the 293 serovars in order to understand the differences in colonization and pathogenicity among 294 serovars colonizing pigs.

\section{Acknowledgments}

296 We gratefully acknowledge funding from SENESCYT-ECUADOR (Secretaría de Educación 297 Superior Ciencia y Tecnología Del Ecuador; Programa de Becas para Docentes Universitarios 298 http://www.educacionsuperior.gob.ec), and would also like to thank Christelle Fablet, from 299 the ANSES Ploufragan Unit of Swine Epidemiology and Welfare Research, for her help in the 300 statistical analysis. 
References

AFNOR 2008. NF U47-102, Animal health analysis methods - Isolation and identification of any Salmonella serotype or of specified Salmonella serotypes among mammals (France).

Berends, B.R., Van Knapen, F., Snijders, J.M., Mossel, D.A., 1997. Identification and quantification of risk factors regarding Salmonella spp. on pork carcasses. Int J Food Microbiol 36, 199-206.

Bonardi, S., 2017. Salmonella in the pork production chain and its impact on human health in the European Union. Epidemiology \& Infection 145, 1513-1526.

Boyen, F., Haesebrouck, F., Maes, D., Van Immerseel, F., Ducatelle, R., Pasmans, F., 2008. Non-typhoidal Salmonella infections in pigs: a closer look at epidemiology, pathogenesis and control. Vet Microbiol 130, 1-19.

Boyen, F., Pasmans, F., Van Immerseel, F., Donne, E., Morgan, E., Ducatelle, R., Haesebrouck, F., 2009. Porcine in vitro and in vivo models to assess the virulence of Salmonella enterica serovar Typhimurium for pigs. Laboratory animals 43, 46-52.

Bugarel, M., Granier, S.A., Bonin, E., Vignaud, M.L., Roussel, S., Fach, P., Brisabois, A., 2012a. Genetic diversity in monophasic $(1,4,[5], 12: 1:-$ and 1,4,[5],12:-:1,2) and in nonmotile (1,4,[5],12:-:-) variants of Salmonella enterica S. Typhimurium. Food Research International 45 1016-1024.

Bugarel, M., Vignaud, M.L., Moury, F., Fach, P., Brisabois, A., 2012b. Molecular identification in monophasic and nonmotile variants of Salmonella enterica serovar Typhimurium. MicrobiologyOpen 1, 481-489.

Cevallos-Almeida, M., Houdayer, C., Rose, V., Bailly, Y., Paboeuf, F., Fablet, C., Denis, M., Kerouanton, A., 2018. Colonization of Pigs Experimentally Infected with a Monophasic Variant of Salmonella Typhimurium. Foodborne Pathog Dis.

Cote, S., Letellier, A., Lessard, L., Quessy, S., 2004. Distribution of Salmonella in tissues following natural and experimental infection in pigs. Can J Vet Res 68, 241-248.

Denis, M., Houard, E., Fablet, A., Rouxel, S., Salvat, G., 2013. Distribution of serotypes and genotypes of Salmonella enterica species in French pig production. Vet Rec 173, 370.

EFSA, ECDC, 2016. The European Union summary report on trends and sources of zoonoses, zoonotic agents and food-borne outbreaks in 2015. EFSA Journal 14(12), 231.

Gosling, R.J., Mueller-Doblies, D., Martelli, F., Nunez-Garcia, J., Kell, N., Rabie, A., Wales, A.D., Davies, R.H., 2018. Observations on the distribution and persistence of monophasic Salmonella Typhimurium on infected pig and cattle farms. Vet Microbiol 227, 90-96.

Ivanek, R., Osterberg, J., Gautam, R., Sternberg Lewerin, S., 2012. Salmonella fecal shedding and immune responses are dose- and serotype- dependent in pigs. PLoS One 7, e34660.

Larsson, J.T., Torpdahl, M., Petersen, R.F., Sorensen, G., Lindstedt, B.A., Nielsen, E.M., 2009. Development of a new nomenclature for Salmonella Typhimurium multilocus variable number of tandem repeats analysis (MLVA). Euro Surveill 14, 1-5.

Le Devendec, L., Jouy, E., Paboeuf, F., de Boisseson, C., Lucas, P., Drider, D., Kempf, I., 2018. Development of a pig infection model with colistin-resistant Escherichia coli. Vet Microbiol 226, 81-88.

Loynachan, A.T., Nugent, J.M., Erdman, M.M., Harris, D.L., 2004. Acute infection of swine by various Salmonella serovars. J Food Prot 67, 1484-1488.

Lynch, H., Arguello, H., Walia, K., Lawlor, P.G., Duffy, G., Gardiner, G.E., Leonard, F.C., 2017. Evaluation of an Alternative Experimental Infection Method, Which Closely 
Mimics the Natural Route of Transmission of Monophasic Salmonella Typhimurium in Pigs. Foodborne Pathog Dis 14, 23-28.

Matiasovic, J., Stepanova, H., Kudlackova, H., Havlickova, H., Sisak, F., Rychlik, I., Chlebova, K., Leva, L., Osvaldova, A., Gebauer, J., Faldyna, M., 2014. Immune response of pigs to Salmonella enterica serovar Derby and Typhimurium infections. Veterinary Microbiology 170, 284-290.

Osterberg, J., Lewerin, S.S., Wallgren, P., 2009. Patterns of excretion and antibody responses of pigs inoculated with Salmonella Derby and Salmonella Cubana. Vet Rec 165, 404408.

Osterberg, J., Lewerin, S.S., Wallgren, P., 2010. Direct and indirect transmission of four Salmonella enterica serotypes in pigs. Acta Vet Scand 52, 1-7.

Osterberg, J., Wallgren, P., 2008. Effects of a challenge dose of Salmonella Typhimurium or Salmonella Yoruba on the patterns of excretion and antibody responses of pigs. Vet Rec 162, 580-586.

Ribot, E.M., Fair, M.A., Gautom, R., Cameron, D.N., Hunter, S.B., Swaminathan, B., Barrett, T.J., 2006. Standardization of pulsed-field gel electrophoresis protocols for the subtyping of Escherichia coli O157:H7, Salmonella, and Shigella for PulseNet. Foodborne Pathog Dis 3, 59-67.

Scherer, K., Szabó, I., Rösler, U., Appel, B., Hensel, A., Nöckler, K., 2008. Time course of infection with Salmonella typhimurium and its influence on fecal shedding, distribution in inner organs, and antibody response in fattening pigs. Journal of Food Protection 71, 699-705.

Shippy, D.C., Bearson, B.L., Holman, D.B., Brunelle, B.W., Allen, H.K., Bearson, S.M.D., 2018. Porcine Response to a Multidrug-Resistant Salmonella enterica serovar I 4,[5],12:i:- Outbreak Isolate. Foodborne Pathog Dis 15, 253-261.

Soyer, Y., Moreno Switt, A., Davis, M.A., Maurer, J., McDonough, P.L., SchoonmakerBopp, D.J., Dumas, N.B., Root, T., Warnick, L.D., Grohn, Y.T., Wiedmann, M., 2009. Salmonella enterica serotype 4,5,12:i:-, an emerging Salmonella serotype that represents multiple distinct clones. J Clin Microbiol 47, 3546-3556.

Switt, A.I., Soyer, Y., Warnick, L.D., Wiedmann, M., 2009. Emergence, distribution, and molecular and phenotypic characteristics of Salmonella enterica serotype 4,5,12:i. Foodborne Pathog Dis 6, 407-415.

Van Cauteren, D., Le Strat, Y., Sommen, C., Bruyand, M., Tourdjman, M., Da Silva, N.J., Couturier, E., Fournet, N., de Valk, H., Desenclos, J.C., 2017. Estimated Annual Numbers of Foodborne Pathogen-Associated Illnesses, Hospitalizations, and Deaths, France, 2008-2013. Emerg Infect Dis 23, 1486-1492.

Van Damme, I., Mattheus, W., Bertrand, S., De Zutter, L., 2018. Quantification of hygiene indicators and Salmonella in the tonsils, oral cavity and rectal content samples of pigs during slaughter. Food Microbiology 71, 120-128.

Vieira-Pinto, M., Temudo, P., Martins, C., 2005. Occurrence of salmonella in the ileum, ileocolic lymph nodes, tonsils, mandibular lymph nodes and carcasses of pigs slaughtered for consumption. J Vet Med B Infect Dis Vet Public Health 52, 476-481.

Vieira-Pinto, M., Tremudo, P., Dominguez, L., Fernandez Garayzabal, J.F., Vela, A.I., Bernardo, F., Lobo, C., Oliveira, M. 2012. Virulence Characterization of Salmonella Typhimurium 1,4,[5],12:i:-, the New Pandemic Strain, In: Anous, B., Gurtler, J.B. (Eds.) Salmonella - Distribution, Adaptation, Control Measures and Molecular Technologies. IntechOpen, 291-312.

Weaver, T., Valcanis, M., Mercoulia, K., Sait, M., Tuke, J., Kiermeier, A., Hogg, G., Pointon, A., Hamilton, D., Billman-Jacobe, H., 2017. Longitudinal study of Salmonella 1,4,[5],12:i:- shedding in five Australian pig herds. Prev Vet Med 136, 19-28. 
399

400

401

402

403
Xu, C., Ren, X., Feng, Z., Fu, Y., Hong, Y., Shen, Z., Zhang, L., Liao, M., Xu, X., Zhang, J., 2017. Phenotypic Characteristics and Genetic Diversity of Salmonella enterica Serotype Derby Isolated from Human Patients and Foods of Animal Origin. Foodborne Pathog Dis 14, 593-599. 
Table 1: Information on the three Salmonella strains used for the experimental design

\begin{tabular}{|c|c|c|c|c|c|}
\hline Serovar (Group) & Strain $\mathbf{N}^{\circ}$ & $\begin{array}{c}\text { Year of } \\
\text { isolation }\end{array}$ & $\begin{array}{l}\text { Source / Production } \\
\text { stage }\end{array}$ & Resistance profile (MIC) $^{2}$ & MLVA Type ${ }^{3}$ \\
\hline S. Derby (SDb) & 07 CR553 & 2007 & $\mathrm{MLN}^{1} /$ fattening & Tetracyclin / Spectinomycin & ST-40 \\
\hline S. Typhimurium (ST) & 07CR095 & 2007 & MLN / fattening & None & $2-10-5-N-112$ \\
\hline $\begin{array}{l}\text { monophasic variant of } \\
S . \text { Typhimurium (mST) }\end{array}$ & S12AK050 & 2012 & Feces / fattening & Tetracyclin & $3-12-8-\mathrm{N}-211$ \\
\hline \multicolumn{6}{|c|}{${ }^{1}$ MLN : mesenteric lymph node; ${ }^{2}$ Minimal inhibitory concentrations (MIC) of antibiotics were determined for all strains using the broth dilution method with a custom } \\
\hline
\end{tabular}


Table 2: Average (Mean) and standard deviation (SD) of fecal excretion (in $\log _{10}$ CFU/g) calculated for Salmonella-positive piglets and Average

7 (Mean) and standard deviation (SD) of serology results (in OD\%) for each sampling day and for all serovars.

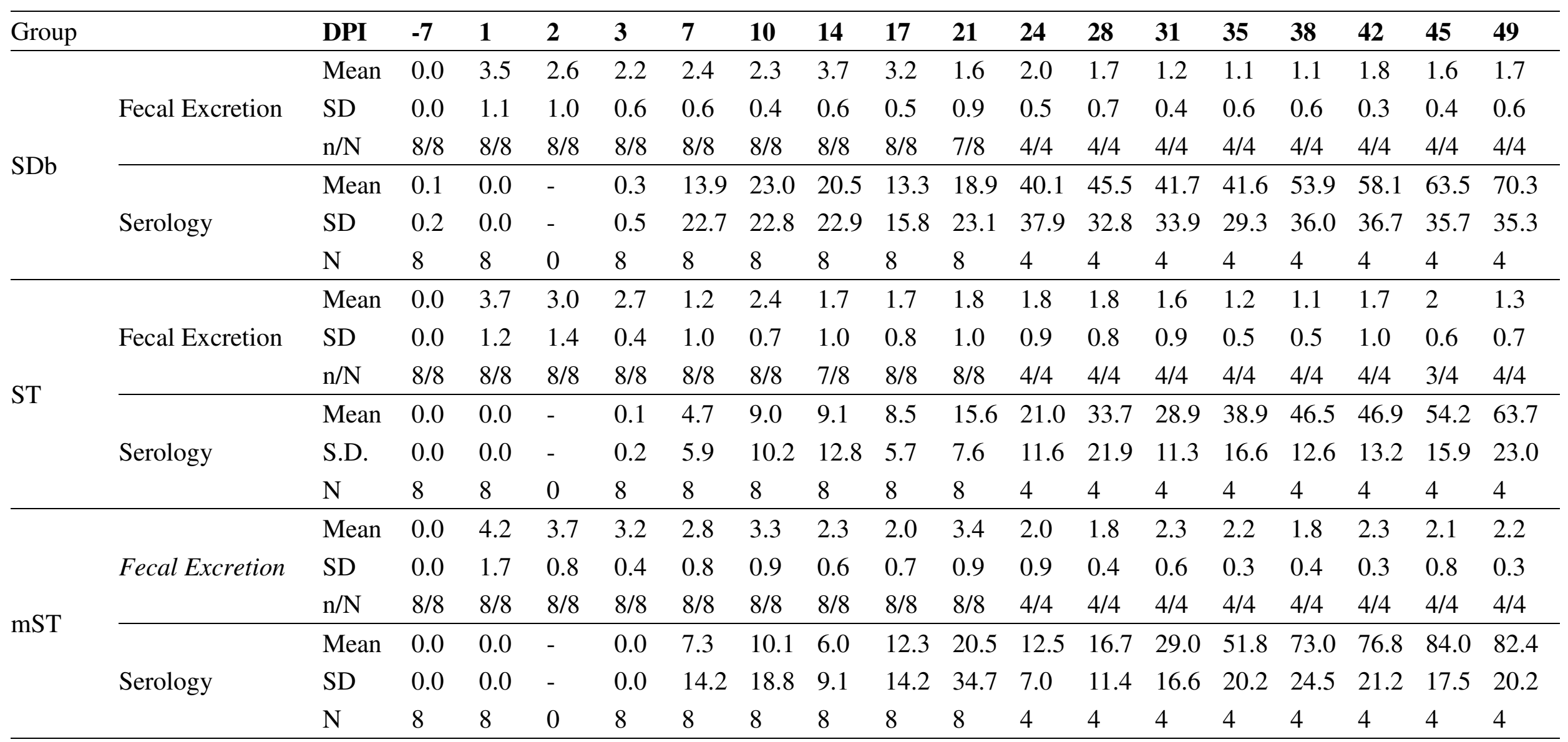

N: number of sampled piglets for each day of sampling (D-7 to D49); n: number of Salmonella-positive piglets. CFU: Colony-Forming Units 
Table 3: Salmonella contamination levels in organs and intestinal contents in $\log _{10}$ CFU/g (mean $\left.\pm S D\right)$ for each inoculated group, at necropsy, on

9 day 21 p.i., on day 49 p.i., and in total.

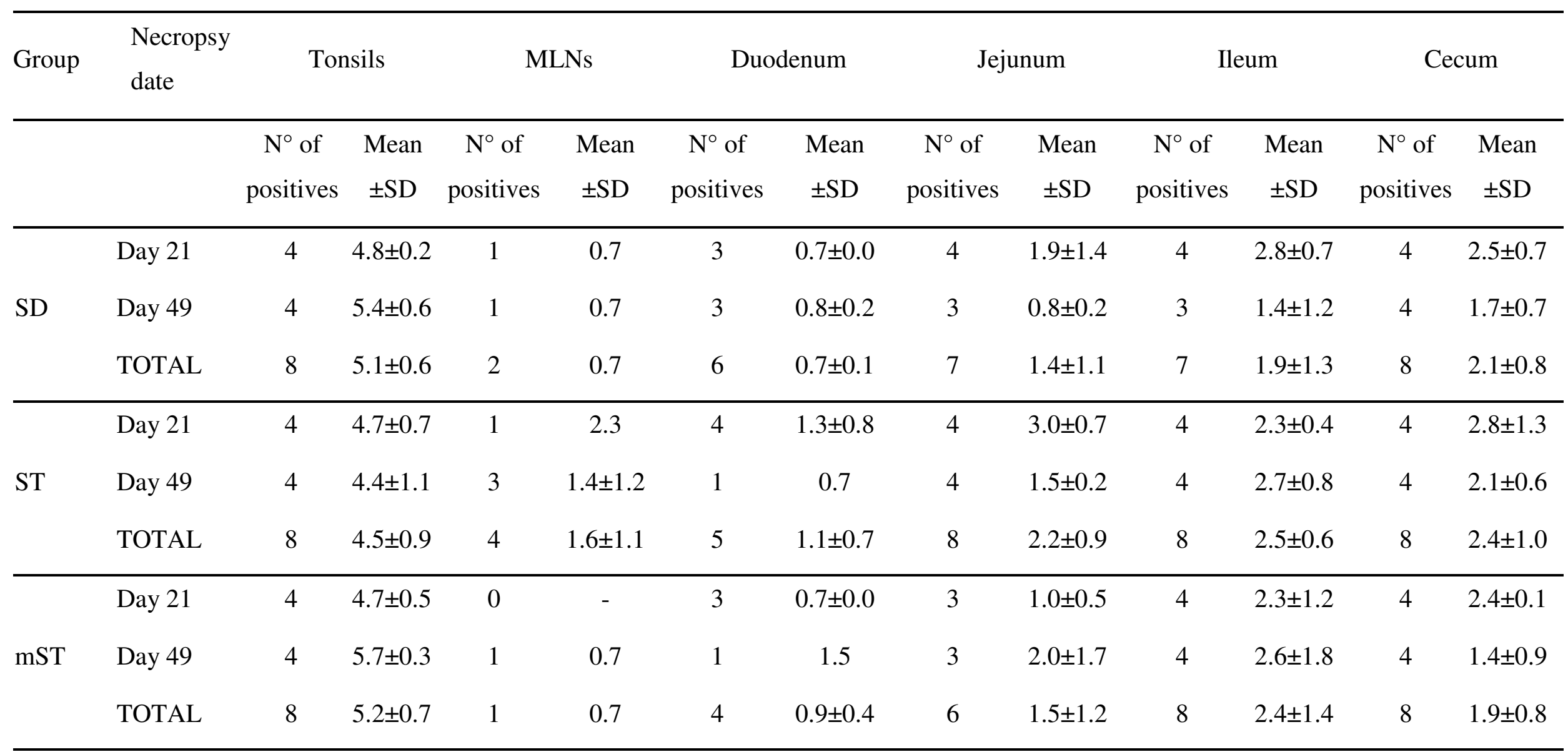

10 MLNs: Mesenteric Lymphatic Nodes, SDb: S. Derby, ST: S. Typhimurium, mST: monophasic S. Typhimurium. SD: Standard deviation 


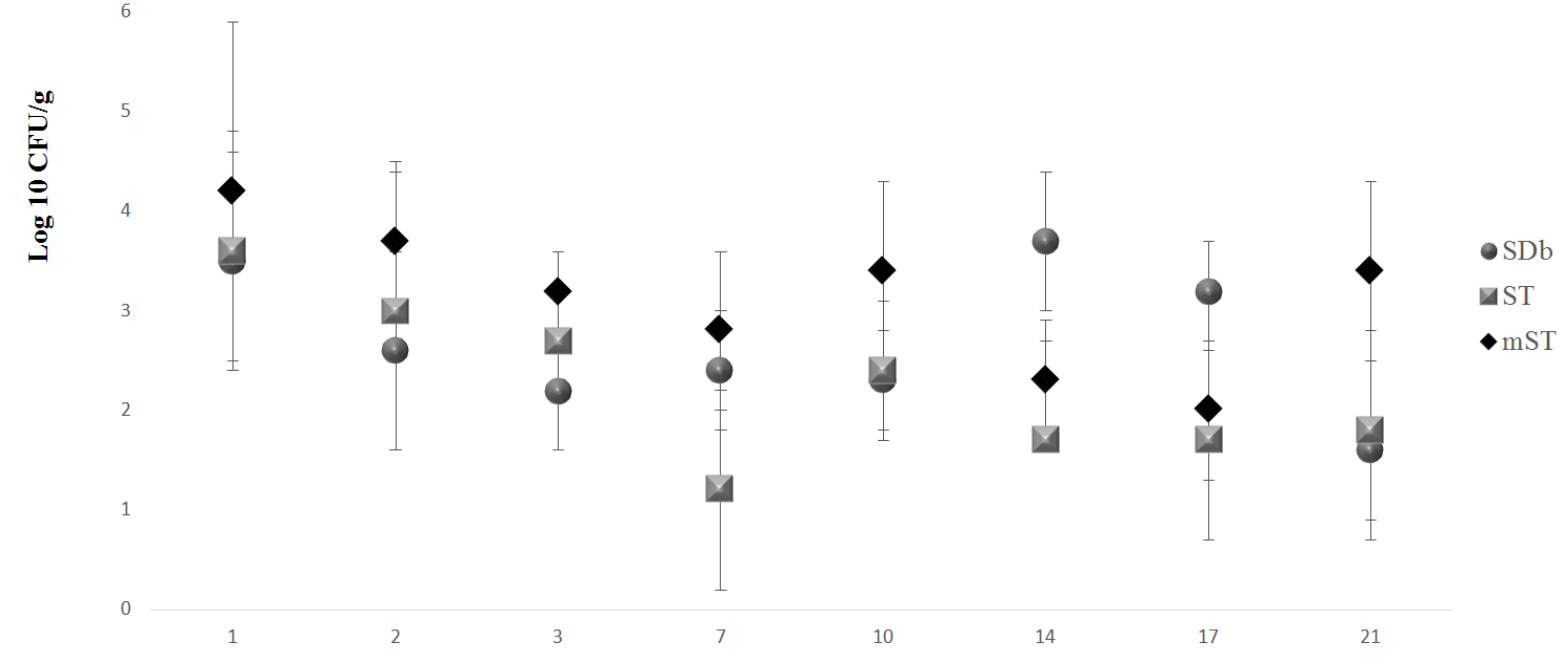

Days

3 Figure 1: Average fecal excretion in $\log _{10}$ UFC/g over 21 days; calculations based on the

4 results of 8 piglets per serovar (except for days 14 and 21, when results are based on 7 piglets

5 for ST and 7 for SD). SDb: S. Derby, ST: S. Typhimurium, mST: monophasic S. Typhimurium 

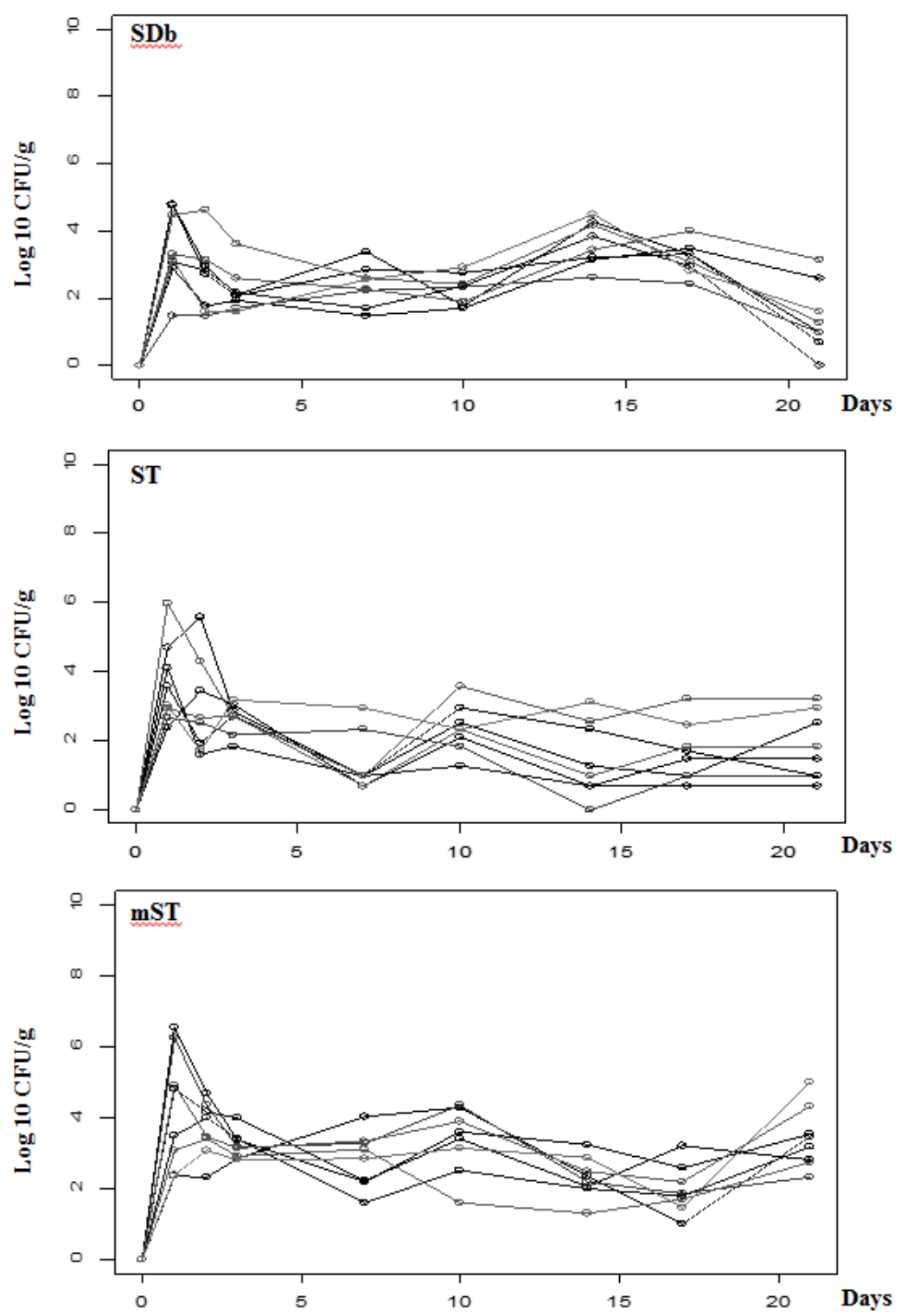

6

$7 \quad$ Figure 2: Kinetics of Salmonella excretion in Log10 UFC/g for the piglets in each group (8

8 piglets per group), over 21 days. Total fecal shedding was calculated from these curves by

9 calculating the cumulative area under the log curve (AULC). SDb: S. Derby, ST: S.

10 Typhimurium, mST: monophasic S. Typhimurium 


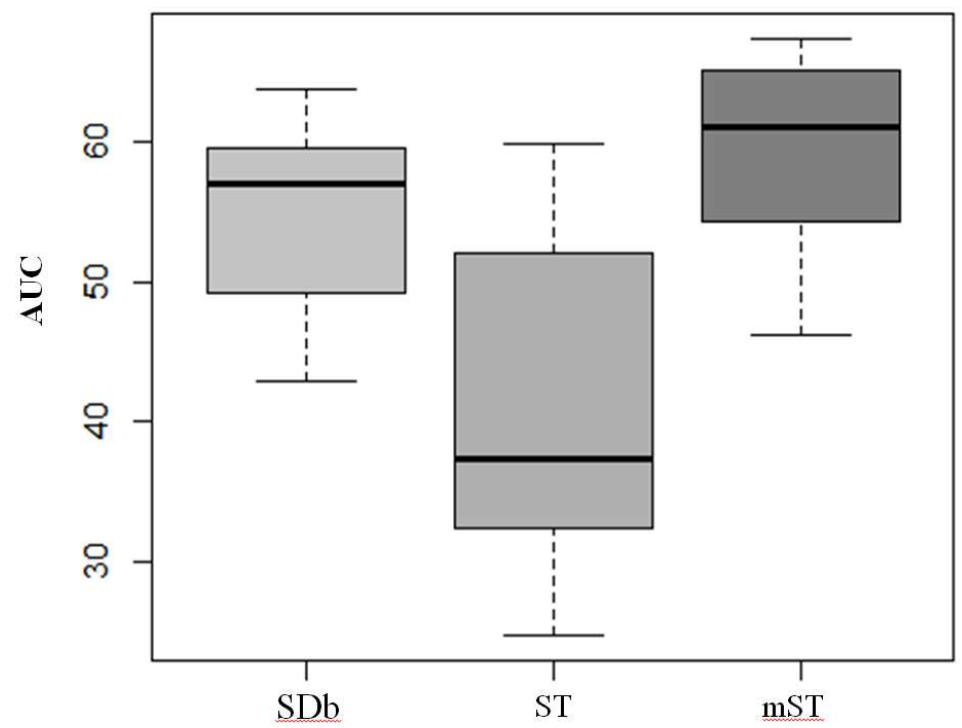

13 Figure 3: Comparison of AULC obtained for each group (21 days). SDb: S. Derby, ST:

14 S. Typhimurium, mST: monophasic S. Typhimurium 

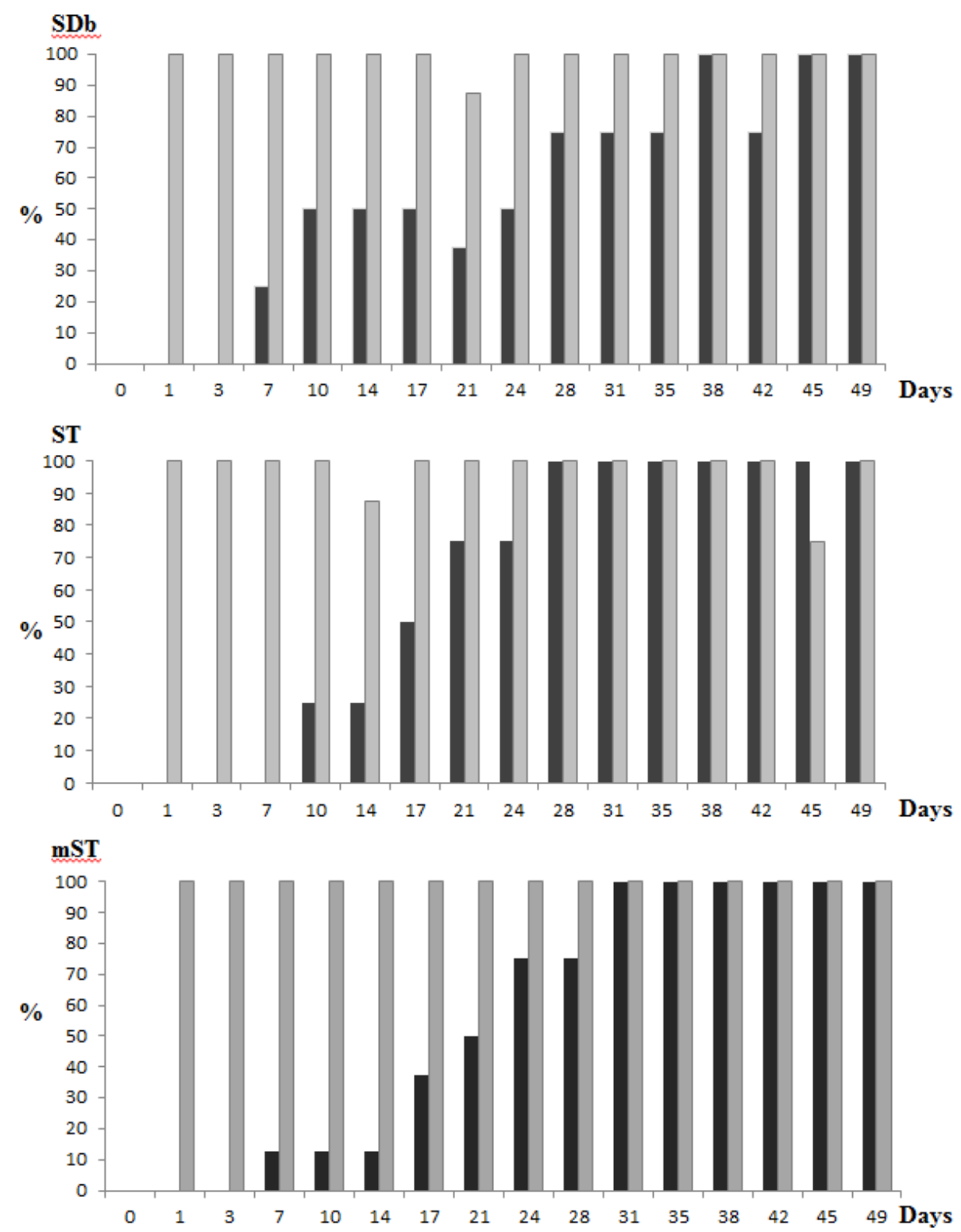

17 Figure 4: Comparison between the percentage (\%) of Salmonella-seroconverted pigs (in

18 black) and Salmonella-shedding pigs (in gray) per day, over the 49 days of the trial. SDb:

19 S. Derby, ST: S. Typhimurium, mST: monophasic S. Typhimurium. 